\title{
Radiology Physics 300 App Review
}

\author{
Ranjit S. Sandhu ${ }^{1}$
}

Published online: 21 December 2015

(C) Society for Imaging Informatics in Medicine 2015

\section{App Specs}

App icon URL: http://is5.mzstatic.com/image/thumb/Purple/ v4/f0/04/cc/f004cc49-e387-c621-306a-8b383405f1a9/ source/100x100bb.jpg

App name: Radiology Physics 300

App developer: Hawki Inc

App developer website: https://itunes.apple.com/us/ developer/hawki-inc/id567122603

App price: $\$ 4.99$

Apple App Store URL: https://itunes.apple.com/us/app/ radiology-physics-300/id600722065? $\mathrm{mt}=8$

Google Play Store URL: N/A

Category: Educational

Tags: \#educational \#radiology \#physics \#works offline \#iOSonly

Works offline: Y

FDA approval: N/A

Promotion code: None

\section{Quick Review}

(1 star: lowest/5 stars: highest)

Overall rating (1-5): 4

Content (1-5): 5

Usability (1-5): 3

Ranjit S. Sandhu

ranjit.s.sandhu@gmail.com

1 Department of Radiology, New York Presbyterian Hospital - Columbia University Medical Center, 622 West 168th St, PB 1-301, New York, NY 10032, USA
Pros Excellent and engaging multiple choice test review of radiology physics representative of the new ABR physics curriculum.

Cons Limited depth of material tested. Limited features. Formatting issues on the iPhone version in portrait orientation decrease usability.

At a glance Easy to use app providing a rapid review of radiology physics with well-written multiple choice questions and sufficient answer explanations.

\section{Full Review}

Introduction Radiology Physics 300 by Hawki Inc is an iOS app designed for radiologists and trainees to review radiology physics. This app is successful in its attempt to address the lack of updated review materials in light of the recent changes to the American Board of Radiology (ABR) examinations.

Purpose/features/content This iOS app available for both iPhone and iPad contains 300 multiple choice questions designed to review the principles of radiology physics with special emphasis on topics now stressed by the American Board of Radiology (ex. imaging artifacts).

Usability The interface is intuitive and straightforward. App response time and scrolling are seamless. Opening the app brings up a prompt requesting the user to select Exam Mode or Answer Mode. Either mode brings up the 300 questions along the left side of the screen starting with question 1. The upper right-hand corner of the screen features a randomization option. Turning randomization on forces you to answer the questions in a random order, but the questions retain their designed number (ex. question \#2 will always be question \#2). 
Next to the randomization feature is a box allowing the user to type a question number to go straight to that question (iPad version only). Exam Mode brings up a 3-h timer along the bottom of the screen that begins counting down immediately. After you select an answer choice, the app prompts you to lock in the answer choice, which can no longer be changed for the remainder of the session. In Answer Mode, locking in the answer choice triggers the display of the answer along the bottom half of the screen before moving on to the next question. Underneath the answer choices is the space reserved for the answer explanation, but if there is an image associated with the question, the image will occupy the space between the answer choices and the answer explanation. If you leave the app but allow it to remain running in the background, you can return to where you left off. If you terminate the app, you have to start all over again. The app works offline since it does not require or utilize an Internet connection after installation.

Good The questions and answers focus on relevant topics and key points with minimal minutia. The breadth of content is excellent and representative of the new physics curriculum designed by the ABR. The questions emphasize conceptual relationships and

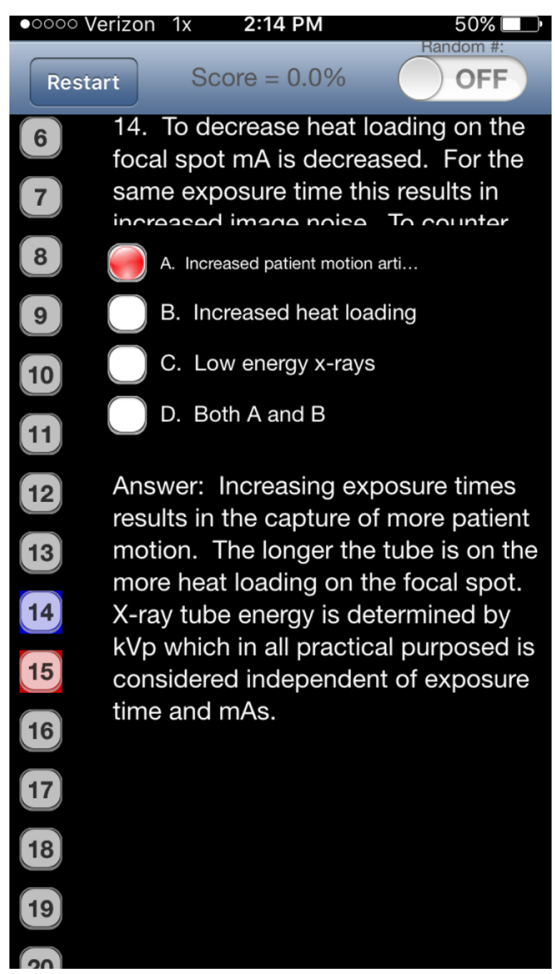

Fig. 1 iPhone version in portrait orientation demonstrates question cutoff (remedied by scrolling). Answer choice A also appears cutoff, which can only be remedied by switching to landscape orientation

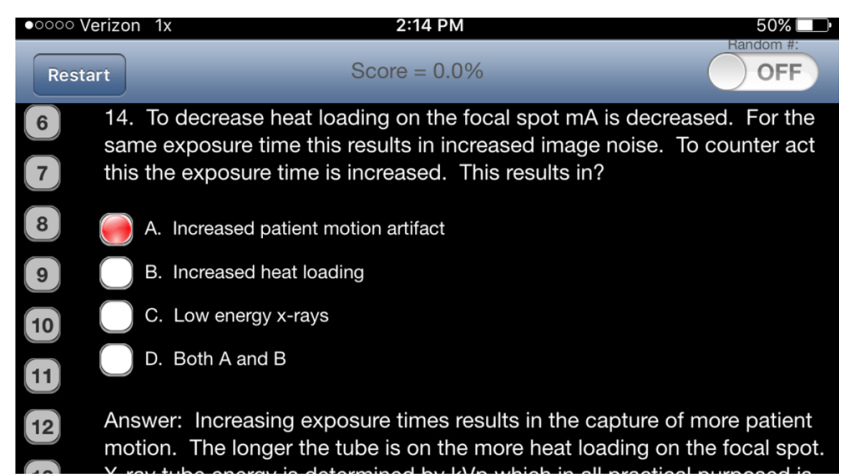

Fig. 2 iPhone version in landscape orientation fixes the cutoff issues seen in portrait orientation

imaging artifacts. The content is presented simply with clear language and concise explanations. The images shown are mostly representative.

Room for improvement The depth of content is lacking since only 300 questions are presented. Increasing the number of questions would more adequately cover the material within the field of radiology physics, but the app has not been updated since 2013. The concise answer explanations make for efficient review, but sometimes the explanations do not discuss why other answer choices are incorrect. No references are provided for further reading. Questions can sometimes appear cutoff since the space reserved for them is smaller than the space reserved for the answer choices, which is a poor utilization of screen space when associated images are not present. This forces

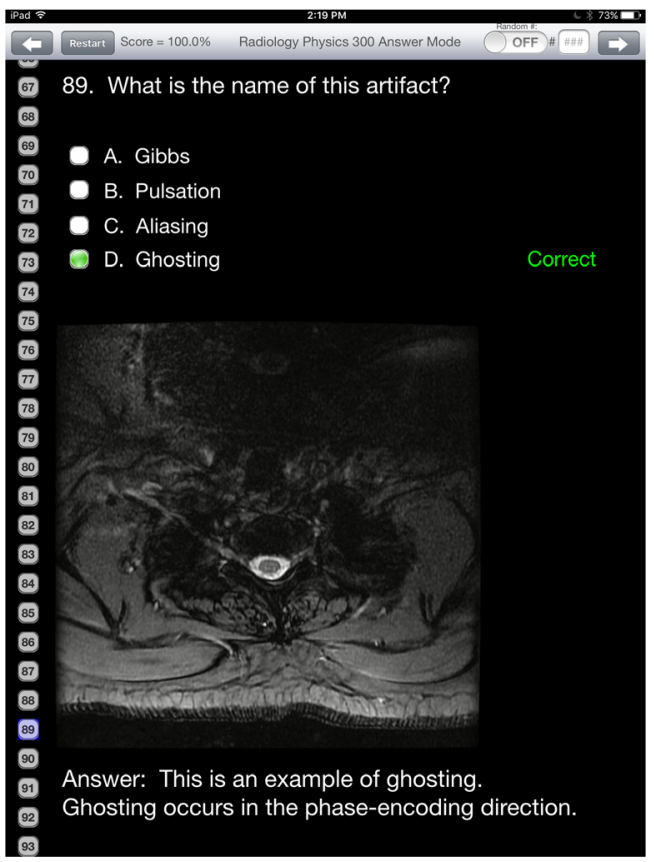

Fig. 3 iPad version in portrait orientation 
the user to scroll down to see the rest of the question for longer questions. This issue also occurs with longer answer choices when using the iPhone version in portrait orientation (Fig. 1), but scrolling is not possible so the iPhone must be placed in landscape orientation to see the answer choices or an iPad can be used instead (Figs. 2 and 3). The inability to pause and resume where you left off between sessions (if the app is terminated) forces the user to remember or jot down which questions were answered/unanswered; this is even more problematic if the randomization feature is used. While the app does keep score of questions answered correctly, this is only for a specific session. All score information is lost once the app is terminated. 